JOURNAL OF RESEARCH of the National Bureau of Standards - A. Physics and Chemistry

Vol. 76A, No. 4, July-August 1972

\title{
Lattice Vibrations of Antiparallel Chain Sheet Structures
}

\author{
Bruno M. Fanconi \\ Institute for Materials Research, National Bureau of Standards, Washington, D.C. 20234
}

(January 21, 1972)

\begin{abstract}
Methods are developed for calculating the normal coordinate vibrations of isolated helical homopolymers and of the antiparallel sheet structures formed by some helical polymers in the solid state. The dynamical equations are expressed in Cartesian displacement coordinates starting from an internal coordinate harmonic force field. As an example of the method the dynamical equations of the honey. comb lattice are derived.
\end{abstract}

Key words: Antiparallel chain sheet structures; honeycomb lattice; lattice vibrations.

\section{Introduction}

An essential feature of many polymeric crystals is layered structures which are held together primarily by interchain hydrogen bonding. Examples of polymeric materials which form this type of structure include some nylons, synthetic polypeptides, and fibrous proteins. Interchain bonding is expected to influence vibrational properties especially in the low frequency region and thereby affect the infrared and Raman spectra, the inelastic neutron scattering, and thermodynamic properties such as the low temperature specific heat.

With the availability of commercial Fourier transform infrared spectrophotometers and low light scattering Raman spectrometers low frequency vibrational studies on polymeric systems are now practical and several such studies have recently appeared in the literature [1-3]. The assignment of vibrational spectra obtained from solid state samples has been based mainly on normal coordinate calculations of isolated chain models [4-7]. Interchain effects have been either totally ignored or treated in part by perturbation methods $[4,8,9]$ because the vibrational secular equation may become quite large. For example, the normal mode frequencies of polyglycine, $\left(\mathrm{CH}_{2} \mathrm{NHCO}\right)_{n}$, in the fully extended conformation, polyglycine I, may by calculated from an isolated chain model with $\mathrm{C}_{2 \mathrm{v}}$ symmetry by solving a secular equation of dimensionality twenty-one. For the more realistic twodimensional pleated sheet model the dimensionality of the secular equation is 84 since four glycine residues comprise the two-dimensional unit cell. To calculate the vibrational frequency as a function of the phase differences (phonon dispersion curves) requires the solution of a secular equation which is twice as large because the matrix elements are, in general, complex.
The phonon dispersion curves are useful for comparisons with the inelastic neutron scattering data $[7,10]$ and with the low temperature specific heat [7].

In this paper a method is introduced for calculating the normal coordinates in terms of the Cartesian displacement coordinates of isolated helical homopolymers. This method is extended to antiparallel sheet structures with four chemical repeat units comprising the two-dimensional unit cell. The method is demonstrated for the honeycomb lattice shown in figure 1 .

\section{Method for Calculating the Normal Coordinates of an Isolated Chain}

The adaption of the Wilson GF method [11] to the calculation of the normal coordinates of helical infinite polymers was first described by Higgs [12] who utilized symmetry adapted internal coordinates. Miyazawa and coworkers [13] extended Higgs' method by defining the

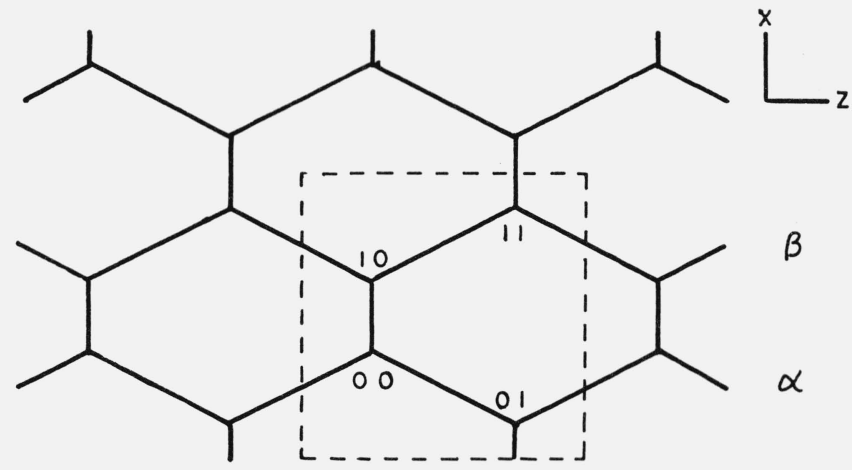

FIGURE 1. Honeycomb lattice unit cell is indicated by dotted lines.

The polymer chains lie along the $z$ axis. The interchain bonds are parallel to the $x$ axis. 
internal coordinates in terms of the helical parameters. This method is rather cumbersome especially for complicated chemical repeat units. Small, et al. [6] have developed a method by which the vibrational secular equation may be set up from the internal coordinates of one chemical repeat unit defined in the Cartesian coordinate system. The dynamical matrix in terms of the internal coordinates in nonhermitian so as to require two separate matrix diagonalizations. Furthermore, there are usually redundant internal coordinates introduced so that the dimensionality of the secular matrix is larger than $3 M$ where $M$ is the number of atoms in the chemical repeat unit. Both of these disadvantages are removed by expressing the vibrational problem in Cartesian displacement coordinates. An expression for the vibrational secular equation for helical polymers in the symmetrized Cartesian displacement coordinate basis set will now be developed following the method devised by Small, et al. [6], for the internal coordinates.

The kinetic energy in terms of the Cartesian displacements is

$$
2 T=\sum_{r=-N / 2+1}^{N / 2} \sum_{j=1}^{3 M} \dot{X}_{j}^{r} m_{j} \dot{X}_{j}^{r}
$$

where $X_{j}^{r}$ is the $j$ th displacement coordinate of the $r$ th chemical repeat unit for a polymer of $N$ total repeat units with reentrant boundary conditions and $m_{j}$ is the mass of the atom undergoing the $X_{j}^{r}$ displacement.

The potential energy assuming harmonic forces is

$$
2 V=\sum_{r, p} \sum_{j, l} X_{j}^{r} f_{j, l}^{r, p} X_{l}^{p}
$$

where

$$
f_{j, l}^{r, p}=\partial^{2} V / \partial X_{j}^{r} \partial X_{l}^{p}
$$

are force constants in terms of Cartesian displacement coordinates.

In matrix notation eqs ( 1 ) and (2) become

$$
2 T=\sum_{r} \underline{\tilde{\mathbf{X}}}^{r} \underline{\underline{\mathbf{M}}} \underline{\dot{\mathbf{X}}}^{r}
$$

and

$$
2 V=\sum_{r, p} \underline{\tilde{\mathbf{X}}}^{r \underline{\mathbf{f}}^{r, p}} \underline{\mathbf{X}}^{p}
$$

where the tilde means the transpose vector. The potential energy, eq ( $4 \mathrm{a})$, may be rewritten as

$$
2 V=\sum_{r} \sum_{s=-k}^{k} \underline{\tilde{\mathbf{X}}}_{\underline{r}}^{\underline{r}^{r}, r+s} \underline{\mathbf{X}}^{r+s}
$$

where the index $s$ labels the neighboring units of the $r$ th unit and $\underline{\underline{\mathbf{f}}}^{r+s}=0$ for $|s|>k$.

The symmetry coordinates, $\underline{\mathbf{X}}(\theta)$, are linear combinations of equivalent coordinates. Unlike the internal coordinates which are invariant of unit location and equivalent, the Cartesian coordinates depend on unit location but may be made to form an equivalent set through the rotational operator $\underline{\underline{H}}^{r}(\psi)$. The matrix
$\underline{\underline{\mathbf{H}}}^{r}(\psi)$ is comprised of $M 3 \times 3$ blocks along the diagonal where each block is of the form

$$
\left(\begin{array}{ccc}
\cos (r \psi) & -\sin (r \psi) & 0 \\
\sin (r \psi) & \cos (r \psi) & 0 \\
0 & 0 & 1
\end{array}\right)
$$

and $\psi$ is the helical angle.

The matrix $\underline{\mathbf{H}}^{r}(\psi)$ rotates the $x, y$ coordinates of the 0th unit into those of the $r$ th unit. The form of transformation given in eq (5) assumes a right-handed helix with helical axis $Z$ and with $r>0$ labeling units reached by clockwise rotations plus translations.

The normalized symmetry coordinates $\underline{X}(\theta)$ are defined as

$$
\underline{\mathbf{X}}(\theta)=1 / \sqrt{N} \sum_{r} \exp (-i r \theta) \underline{\underline{\mathbf{H}}}^{-r}(\psi) \underline{\mathbf{X}}^{r} .
$$

Solving eq (6) for $\underline{\mathrm{X}}^{r}$ and substituting into eq (4b) yields

$$
\begin{aligned}
& 2 V=\sum_{r, s} \sum_{\theta, \theta^{\prime}} \exp \left[\operatorname{ir}\left(\theta^{\prime}-\theta\right)\right] \\
& \underline{\tilde{\mathbf{X}}}(\theta) \underline{\underline{\mathbf{H}}}^{-r}(\psi) \underline{\underline{\mathbf{f}}}^{r, r+s} \underline{\underline{\mathbf{H}}}^{r}(\psi) \underline{\underline{\mathbf{H}}}^{s}(\psi) \underline{\mathbf{X}}\left(\theta^{\prime}\right) \exp \left(i s \theta^{\prime}\right)
\end{aligned}
$$

From the definition of the transformation matriy $\underline{\underline{\mathbf{H}}}^{r}(\psi)$ and the covariance of $\underline{\underline{\mathbf{f}}}^{r, r+s}$ it follows that

$$
\underline{\underline{\mathbf{H}}}^{-r}(\psi) \underline{\underline{\mathbf{f}}}^{r, r+s} \underline{\underline{\mathbf{H}}}^{r}(\psi)=\underline{\underline{\mathbf{f}}}^{0, s} .
$$

With this result eq (7) becomes

$$
2 V=\sum_{s, \theta} \underline{\tilde{\mathbf{X}}}(\theta) \underline{\underline{\mathbf{f}}}^{0, s} \underline{\underline{\mathbf{H}}}^{s}(\psi) \underline{\mathbf{X}}(\theta) \exp (i s \theta)
$$

The potential energy may also be expressed in the internal coordinate basis set as

$$
2 V=\sum_{t, q} \underline{\underline{\mathbf{R}}}^{t} \underline{\mathbf{F}}^{t, q} \underline{\underline{\mathbf{R}}}^{q}
$$

where $\underline{\mathbf{R}}^{q}$ is the column vector of internal coordinates in the $q$ th chemical repeat unit and

$$
F_{i, j}^{t, q}=\partial^{2} V / \partial R_{i}^{t} \partial R_{j}^{q}
$$

are the internal coordinate force constants.

The internal coordinates are defined through t] B matrices [6],

$$
\underline{\mathbf{R}}^{q}=\sum_{p} \underline{\underline{\mathbf{B}}}^{q, p} \underline{\mathbf{X}}^{p}
$$

so that eq (9) becomes

$$
2 V=\sum_{t, q} \sum_{p, r} \underline{\tilde{\mathbf{X}}}^{r \underline{\underline{\mathbf{B}}}^{t, r}} \underline{\underline{\mathbf{F}}}^{t, q} \underline{\underline{\mathbf{B}}}^{q, p} \underline{\underline{\mathbf{X}}}^{p}
$$

which yields upon comparison with eq (4a)

$$
\underline{\underline{\mathbf{f}}}^{r, p}=\sum_{t, q} \underline{\tilde{\mathbf{B}}}^{t, r} \underline{\underline{\mathbf{F}}}^{t, q} \underline{\underline{\mathbf{B}}}^{q, p} .
$$


Utilizing the expression [6]

$$
\underline{\underline{\mathbf{B}}}^{q, p}=\underline{\underline{\mathbf{B}}}^{0, p-q} \underline{\underline{\mathbf{H}}}^{-q}(\psi)
$$

and the fact that $\underline{\underline{\mathbf{F}}}^{t, q}=\underline{\underline{\mathbf{F}}}^{0, q-t}$ we have

$$
\underline{\underline{\mathbf{f}}}^{0, s}=\sum_{t, r} \underline{\underline{\mathbf{H}}}^{t}(\psi) \underline{\underline{\mathbf{B}}}^{0,-t} \underline{\underline{\mathbf{F}}}^{0, r} \underline{\underline{\mathbf{B}}}^{0, s-t-r} \underline{\underline{\mathbf{H}}}(\psi)^{-(t+r)}
$$

where the summations are over positive and negative values. We find by substitution of eq (10) into eq (8) the potential energy in terms of the Cartesian displacement symmetry coordinates and the internal coordinate force matrix:

$$
\begin{aligned}
2 V=\sum_{\theta} \sum_{r, s, t} \underline{\tilde{\mathbf{X}}}_{(\theta)} \underline{\underline{\mathbf{H}}}^{-t}(\psi) \underline{\underline{\tilde{\mathbf{B}}}}^{s, t} \exp (-i t \theta) \underline{\underline{\mathbf{F}}}^{o, r} \\
\exp (i r \theta) \underline{\underline{\mathbf{B}}}^{0, s} \underline{\underline{\mathbf{H}}}^{s}(\psi) \exp (i s \theta) \underline{\mathbf{X}}(\theta) .
\end{aligned}
$$

Defining

$$
\underline{\underline{\mathbf{B}}}(\theta)=\sum_{s} \exp (i s \theta) \underline{\underline{\mathbf{B}}}^{0, s} \underline{\underline{\mathbf{H}}}^{s}(\psi)
$$

and

$$
\underline{\underline{\mathbf{F}}}(\theta)=\sum_{r} \exp (i r \theta) \underline{\underline{\mathbf{F}}}^{0, r} .
$$

Equation (11) becomes

$$
2 V=\sum_{\theta} \underline{\tilde{\tilde{\mathbf{X}}}}(\theta) \underline{\underline{\tilde{\mathbf{B}}}}(\theta) \underline{\underline{\mathbf{F}}}(\theta) \underline{\underline{\mathbf{B}}}(\theta) \underline{\underline{X}}(\theta) .
$$

The kinetic energy, eq (3), becomes in the Cartesian symmetry coordinates

$$
2 T=\sum_{\theta} \underline{\dot{\mathbf{X}}}(\theta) \underline{\underline{\mathbf{M}}} \underline{\dot{\mathbf{X}}}(\theta)
$$

or in the conjugate momenta

$$
2 T=\sum_{\theta} \underline{\tilde{\mathbf{P}}}(\theta) \underline{\underline{\mathbf{M}}}^{-1} \underline{\mathbf{P}}(\theta) .
$$

From eqs (14 and 15), we find through Hamilton's equations the eigenvalue equation

$$
\underline{\underline{\mathbf{M}}}^{-1} \underline{\underline{\mathbf{B}}}(\theta) \underline{\underline{\mathbf{F}}}(\theta) \underline{\underline{\mathbf{B}}}(\theta) \underline{\underline{\mathbf{L}}}_{X}(\theta)=\underline{\underline{\mathbf{L}}}_{X}(\theta) \underline{\underline{\Lambda}}(\theta)
$$

where $\Lambda(\theta)$ is the diagonal matrix of the squares of the eigenfrequencies and $L_{X}$ is the matrix of eigenvectors. Equation (16) may be symmetrized in the form

$$
\underline{\underline{\mathbf{M}}}^{-1 / 2} \underline{\underline{\underline{\mathbf{B}}}}(\theta) \underline{\underline{\mathbf{F}}}(\theta) \underline{\underline{\mathbf{B}}}(\theta) \underline{\underline{\mathbf{M}}}^{-1 / 2} \underline{\underline{\mathbf{L}}}_{X}^{\prime}(\theta)=\underline{\underline{\mathbf{L}}}_{X}^{\prime}(\theta) \underline{\underline{\Lambda}}(\theta)
$$

where $\underline{\underline{\mathbf{L}}}_{X}^{\prime}(\bar{\theta})=\underline{\underline{M}}^{1 / 2} \underline{\underline{\mathbf{L}}}_{X}$ is the matrix of eigenvectors in the mass-reduced symmetry coordinates.

The form of eq (17) is identical to that given previcusly [14] for translational symmetry (i.e. $\underline{\underline{\mathbf{H}}}^{r}(\psi)$ is the unit matrix). The essential feature in this derivation is that the dimensionality of $\underline{\underline{\mathbf{B}}}(\theta)$ equals the number of independent coordinates in the chemical repeat unit and that only the internal coordinates of one chemical repeat unit in terms of Cartesian displacement coordinates need be specified.

\section{Two-Dimensional Lattice of Antiparallel Chains}

Consider a two dimensional lattice, figure 1 , in the $X, Z$ plane with two chains passing through the unit cell and helical axes parallel to $Z$. The helical angle, $\psi$, is $180^{\circ}$ and there is a two-fold screw rotation axis along $X$. The latter symmetry operation reverses the sense of the two chains to produce the antiparallel structure. The translational unit cell contains four chemical repeat units numbered $(0,0),(0,1)$ in the $\alpha$ chain and $(1,0),(1,1)$ in the $\beta$ chain.

The four units are related by the two-fold screw axes along $Z$ and $X$. For the two-dimensional lattice we have [14]

where

$$
\underline{\underline{\mathbf{f}}}(\phi, \theta)=\underline{\underline{\tilde{\mathbf{B}}}}(\phi, \theta) \underline{\underline{\mathbf{F}}}(\phi, \theta) \underline{\underline{\mathbf{B}}}(\phi, \theta)
$$

$$
\begin{aligned}
& \underline{\underline{\mathbf{B}}}(\phi, \theta)=\sum_{r, s} \underline{\underline{\mathbf{B}}}^{r, s} \exp [i(r \phi+s \theta)] \\
& \underline{\underline{\mathbf{F}}}(\phi, \theta)=\sum_{r, s} \underline{\underline{\mathbf{F}}}^{r, s} \exp [i(r \phi+s \theta)]
\end{aligned}
$$

with

$$
\begin{aligned}
\phi & =\underline{\mathbf{k}}_{X} \cdot \underline{\mathbf{t}}_{X} \\
\theta & =\underline{\mathbf{k}}_{Z} \cdot \underline{\mathbf{t}}_{Z} .
\end{aligned}
$$

The quantities $\mathbf{t}_{X}, \mathbf{t}_{Z}$ are the lattice vectors directed along the $X$ and $Z$ axes and $\mathbf{k}_{X}, \mathbf{k}_{Z}$ are the wavevectors. The quantities $\phi$ and $\theta$ are the phase differences between identical displacements in neighboring unit cells. The dimensionality of the matrix $\mathbf{f}(\phi, \theta)$ is $3 m \times 3 m$ where $m$ is the number of atoms in the twodimensional unit cell whereas the dimensionality of $\underline{\mathbf{F}}(\phi, \theta)$ equals the number of internal coordinates $\overline{\bar{w}}$ hich is usually greater than $3 m$ since redundant coordinates are included.

The basis set used to express the quantities in eq (18) are linear combinations of equivalent chemical repeat units:

$$
\begin{aligned}
\underline{\mathbf{X}}_{j, l}(\phi, \theta)=1 / \sqrt{N_{X} N_{Z}} \sum_{r=0}^{N_{X}-1} \sum_{t=0}^{N_{Z}-1} \\
\quad \exp [-i(r \phi+t \theta)] \mathbf{X}_{j, l}^{r, t}
\end{aligned}
$$

where $j, l=0,1$ label the chemical repeat unit and $r, t$ the unit cell. The symmetry coordinates, eq (19), may be related to those of eq (6) as follows. The chemical repeat units are renumbered so that $\underline{\mathbf{X}}^{p, q}$ equals $\mathrm{X}_{j}^{(p-j) / 2,(q-l) / 2}$ with $j, l=0$ or 1 for $p, q$ even or odd. A generalization of eq (6) yields the symmetry coordinates

$$
\begin{gathered}
\underline{\mathbf{X}}\left(\phi^{\prime}, \theta^{\prime}\right)=1 / 2 \sqrt{N_{X} N_{Z}} \sum_{p=0}^{2 N_{X}-1} \sum_{q=0}^{2 N_{Z}-1} \\
\exp \left[-i\left(p \phi^{\prime}+q \theta^{\prime}\right) \underline{\underline{\mathbf{H}}}^{-p,-q} \underline{\mathbf{X}}^{p, q}\right.
\end{gathered}
$$


where $\underline{\underline{\mathbf{H}}}^{p, q}$ is the transformation matrix representing $p 2$-fold rotations about $X$ and $q 2$-fold rotations about $Z$. The matrix $\underline{\underline{\mathbf{H}}}^{p, q}$ is comprised of $m 3 \times 3$ blocks with each block given by

$$
\left(\begin{array}{ccc}
(-1)^{q} & 0 & 0 \\
0 & (-1)^{q+p} & 0 \\
0 & 0 & (-1)^{p}
\end{array}\right) .
$$

Using the relationship between $\underline{\mathbf{X}}^{p, q}$ and $\underline{\mathbf{X}}_{j, l}^{r, t}$, eq (20) may be rewritten as

$$
\begin{aligned}
\underline{\mathbf{X}}\left(\phi^{\prime}, \theta^{\prime}\right)=1 / 2 \sqrt{N_{X} N_{Z}}\left\{\sum_{p, \text { even }} \sum_{q, \text { even }} \exp \left[-i\left(p \phi^{\prime}+q \theta^{\prime}\right)\right] \underline{\mathbf{X}}_{0,0}^{/ 2, q / 2}\right. \\
\quad+\sum_{p, \text { even }} \sum_{q, \text { odd }} \exp \left[-i\left(p \phi^{\prime}+q \theta^{\prime}\right)\right] \underline{\underline{\mathbf{H}}}^{0,1} \underline{\mathbf{X}}_{0,1}^{p / 2,(q-1) / 2} \\
\quad+\sum_{p, \text { odd }} \sum_{q, \operatorname{even}} \exp \left[-i\left(p \phi^{\prime}+q \theta^{\prime}\right)\right] \underline{\underline{\mathbf{H}}}^{1,0} \underline{\mathbf{X}}_{1,0}^{(p-1) / 2, q / 2} \\
\left.\quad+\sum_{p, \text { odd }} \sum_{q, \text { odd }} \exp \left[-i\left(p \phi^{\prime}+q \theta^{\prime}\right)\right] \underline{\underline{\mathbf{H}}}^{1,1} \underline{\mathbf{X}}_{1,1}^{(p-1) / 2,(q-1) / 2}\right\}
\end{aligned}
$$

$$
\begin{aligned}
\mathbf{X}\left(\phi^{\prime}, \boldsymbol{\theta}^{\prime}\right)=1 / 2\left[\mathbf{X}_{0,0}\left(2 \phi^{\prime}, 2 \theta^{\prime}\right)+\right. & \underline{\underline{\mathbf{H}}}^{0,1} \underline{\mathbf{X}}_{0,1}\left(2 \phi^{\prime}, 2 \theta^{\prime}\right) \exp \left(-i \theta^{\prime}\right) \\
& \left.+\underline{\mathbf{H}}^{1,0} \underline{\mathbf{X}}_{1,0}\left(2 \phi^{\prime}, 2 \theta^{\prime}\right) \exp \left(-i \phi^{\prime}\right)+\underline{\underline{\mathbf{H}}}^{1,1} \underline{\mathbf{X}}_{1,1}\left(2 \phi^{\prime}, 2 \theta^{\prime}\right) \exp \left(-i\left(\theta^{\prime}+\phi^{\prime}\right)\right)\right] .
\end{aligned}
$$

Functions orthogonal to $\underline{\mathbf{X}}(\phi, \theta)$ are $\underline{\mathbf{X}}(\phi, \theta+\pi), \underline{\mathbf{X}}(\phi+\pi, \theta)$. and $\underline{\mathbf{X}}(\phi+\pi, \theta+\pi)$ which are related to those of eq (19)

$$
\left[\begin{array}{l}
\mathbf{X}(\phi, \theta) \\
\mathbf{X}(\phi, \theta+\pi) \\
\mathbf{X}(\phi+\pi, \theta) \\
\mathbf{X}(\phi+\pi, \theta+\pi)
\end{array}\right]=\frac{1}{2}\left[\begin{array}{rrrr}
\mathbf{I} & e^{-i \theta} \underline{\underline{\mathbf{H}}}^{o, 1} & e^{-i \theta} \underline{\underline{\mathbf{H}}}^{1,0} & e^{-i(\phi+\theta)} \underline{\underline{\mathbf{H}}}^{1,1} \\
\mathbf{I} & -e^{-i \theta} \underline{\underline{\mathbf{H}}}^{0,1} & e^{-i \phi} \underline{\underline{\mathbf{H}}}^{1,0} & -e^{-i(\phi+\theta)} \underline{\underline{\mathbf{H}}}^{1,1} \\
\mathbf{I} & e^{-i \theta} \underline{\underline{\mathbf{H}}}^{0,1} & -e^{-i \phi} \underline{\underline{\mathbf{H}}}^{1,0} & -e^{-i(\phi+\theta)} \underline{\underline{\mathbf{H}}}^{1,1} \\
\mathbf{I} & -e^{-i \theta} \underline{\underline{\mathbf{H}}}^{0,1} & -e^{-i \phi} \underline{\underline{\mathbf{H}}}^{1,0} & e^{-i(\phi+\theta)} \underline{\underline{\mathbf{H}}}^{1,1}
\end{array}\right]\left[\begin{array}{l}
\underline{\mathbf{X}}_{0,0}(2 \phi, 2 \theta) \\
\underline{\mathbf{X}}_{0,1}(2 \phi, 2 \theta) \\
\underline{\mathbf{X}}_{1,0}(2 \phi, 2 \theta) \\
\underline{\mathbf{X}}_{1,1}(2 \phi, 2 \theta)
\end{array}\right]
$$

or

$$
\left[\begin{array}{l}
\underline{\mathbf{x}}(\phi, \theta) \\
\underline{\mathbf{x}}(\phi, \theta+\pi) \\
\underline{\mathbf{x}}(\phi+\pi, \theta) \\
\underline{\mathbf{x}}(\phi+\pi, \theta+\pi)
\end{array}\right]=\underline{\underline{\mathbf{U}}(\phi, \theta)}\left[\begin{array}{l}
\underline{\mathbf{X}}_{0,0}(2 \phi, 2 \theta) \\
\underline{\mathbf{x}}_{1,0}(2 \phi, 2 \theta) \\
\underline{\mathbf{x}}_{1,1}(2 \phi, 2 \theta)
\end{array}\right]
$$

where I is the identity matrix.

We now derive an expression similar to eq (22) for the internal coordinates so that $\underline{\underline{\mathbf{f}}}(\phi, \theta)$. eq (18). may be transformed to the new symmetry coordinates. It is convenient to list the internal symmetry coordinates of the lattice as $\left[\underline{\mathbf{R}}_{0,0}(\phi, \theta), \underline{\mathbf{R}}_{0,1}(\phi, \theta), \underline{\mathbf{R}}_{\alpha}(\phi . \theta), \underline{\mathbf{R}}_{1,0}(\phi, \theta), \underline{\mathbf{R}}_{1,1}(\phi, \theta), \underline{\mathbf{r}}_{\beta}(\phi, \theta)\right]$ where $\underline{\mathbf{R}}_{j, l}(\phi, \theta)$ are intrachain coordinates of the $j, l$ repeat unit. $\underline{\mathbf{r}}_{\alpha}(\phi, \theta)$ and $\underline{\mathbf{r}}_{\beta}(\phi, \theta)$ are interchain coordinates associated with the $\alpha$ and $\beta$ chain respectively. In a manner analogous to that which lead to eq (22) we find 


$\left[\begin{array}{l}\underline{\mathbf{R}}(\phi, \theta) \\ \underline{\mathbf{R}}(\phi, \theta+\pi) \\ \underline{\mathbf{r}}(\phi, \theta) \\ \underline{\underline{\mathbf{R}}}(\phi+\pi, \theta) \\ \underline{\mathbf{R}}(\phi+\pi, \theta+\pi) \\ \underline{\mathbf{r}}(\phi+\pi, \theta)\end{array}\right]=\frac{1}{2}\left[\begin{array}{llllll}1 & e^{-i \theta} & 0 & e^{-i \phi} & e^{-i(\phi+\theta)} & 0 \\ 1 & -e^{i \theta} & 0 & e^{-i \phi} & e^{-i(\phi+\theta)} & 0 \\ 0 & 0 & \sqrt{2} & 0 & 0 & \sqrt{2} e^{-i \theta} \\ 1 & e^{-i \theta} & 0 & -e^{-i \phi} & -e^{-i(\phi+\theta)} & 0 \\ 1 & -e^{i \theta} & 0 & -e^{-i \phi} & e^{-i(\phi+\theta)} & 0 \\ 0 & 0 & \sqrt{2} & 0 & 0 & -\sqrt{2} e^{-i \theta}\end{array}\right]\left[\begin{array}{l}\underline{\mathbf{R}}_{0,0}(2 \phi, 2 \theta) \\ \underline{\mathbf{R}}_{0,1}(2 \phi, 2 \theta) \\ \underline{\mathbf{r}}_{\alpha}(2 \phi, \theta) \\ \underline{\mathbf{R}}_{1,0}(2 \phi, 2 \theta) \\ \underline{\mathbf{R}}_{1,1}(2 \phi, 2 \theta)\end{array}\right]$

$$
=\underline{=}(\phi, \theta)\left[\begin{array}{l}
\underline{\mathbf{R}}_{0,0}(2 \phi, 2 \theta) \\
\underline{\mathbf{R}}_{0,1}(2 \phi, 2 \theta) \\
\underline{\mathbf{r}}_{\alpha}(2 \phi, \theta) \\
\underline{\mathbf{R}}_{1,0}(2 \phi, 2 \theta) \\
\underline{R}_{1,1}(2 \phi, 2 \theta) \\
\underline{\mathbf{r}}_{\beta}(2 \phi, \theta)
\end{array}\right]
$$

The $\underline{\underline{\mathbf{H}}}^{p, q}$ matrices do not enter into $\underline{\underline{\mathbf{W}}}(\phi, \theta)$ since the internal coordinates are invariant to position within the unit cell. Using eqs (23). (25). $\underline{\underline{\mathbf{f}}}(\phi, \theta)$ is transformed to

where

$$
\underline{\underline{\mathbf{f}}}^{\prime}(\phi, \theta)=\underline{\underline{\mathbf{B}}}^{\prime}(\phi, \theta) \underline{\underline{\mathbf{F}}}^{\prime}(\phi, \theta) \underline{\underline{\mathbf{B}}}^{\prime}(\phi, \theta)
$$

and

$$
\underline{\underline{\mathbf{B}}}^{\prime}(\phi, \theta)=\underline{\underline{\mathbf{W}}}(\phi, \theta) \underline{\underline{\mathbf{B}}}(2 \phi, 2 \theta) \underline{\underline{\mathbf{U}}}(\phi, \theta)
$$

$$
\underline{\underline{\mathbf{F}}}^{\prime}(\phi, \theta)=\underline{\underline{\mathbf{W}}}(\phi, \theta) \underline{\underline{\mathbf{F}}}(2 \phi, 2 \theta) \underline{\underline{\mathbf{U}}}(\phi, \theta) .
$$

In the absence of interchain interactions $\underline{\underline{f}}^{\prime}(\phi, \theta)$ consists of four noninteracting blocks. two of which correspond to intrachain phase difference $\theta$ and the other two to $\theta+\pi$. It will be shown that with certain types of interchain interactions $\underline{\underline{\mathbf{f}}}^{\prime}(\phi, \theta)$ contains nonzero elements only between the blocks $(\phi, \theta)$ and $(\phi+\pi, \theta+\pi)$ and between $(\phi, \theta+\pi),(\phi+\pi, \theta)$ so that the dimensionality of the dynamical matrix has been reduced by a factor of two.

Let

$$
\underline{\underline{\mathbf{B}}}_{i}^{\prime}(\phi, \theta)=1 / \sqrt{2}\left[\begin{array}{cc}
1 & e^{-i \phi} \\
1 & -e^{-i \phi}
\end{array}\right]\left[\begin{array}{c}
\underline{\underline{\mathbf{B}}}_{i}^{\alpha}(2 \phi, 2 \theta) \\
\underline{\underline{\mathbf{B}}}_{i}^{\beta}(2 \phi, 2 \theta)
\end{array}\right] \underline{\underline{\mathbf{U}}(\phi, \theta)}
$$

where $\underline{\underline{B}}_{i}^{\alpha}(2 \phi, 2 \theta), B_{i}^{\beta}(2 \phi, 2 \theta)$ are the $B$ matrices associated with coordinates $\underline{\mathbf{r}}_{\alpha}, \underline{\mathbf{r}}_{\beta}$ respectively. These $B$ matrices may be expanded in terms of contributions from each of the four residues in the unit cell. 
Letting,

$$
\left[\begin{array}{l}
\underline{\underline{\mathbf{B}}}_{i}^{\alpha}(2 \phi, 2 \theta) \\
\underline{\underline{\mathbf{B}}}_{i}^{\beta}(2 \phi, 2 \theta)
\end{array}\right]=\left[\begin{array}{llll}
\underline{\underline{\mathbf{B}}}_{0,0}^{\alpha} & \underline{\underline{\mathbf{B}}}_{0,1}^{\alpha} & \underline{\underline{\mathbf{B}}}_{1,0}^{\alpha} & \underline{\underline{\mathbf{B}}}_{1,1}^{\alpha} \\
\underline{\underline{\mathbf{B}}}_{0,0}^{\beta} & \underline{\underline{\mathbf{B}}}_{0,1}^{\beta} & \underline{\underline{\mathbf{B}}}_{1,0}^{\beta} & \underline{\underline{\mathbf{B}}}_{1,1}^{\beta}
\end{array}\right]
$$

we find upon substitution of eq (28)

$$
\underline{\underline{\mathbf{B}}}^{\alpha}(\phi, \theta)=\underline{\underline{\mathbf{B}}}_{i}^{\alpha}(2 \phi, 2 \theta) \underline{\underline{\tilde{\mathbf{U}}}}(\phi, \theta)
$$

$$
\underline{\underline{\mathbf{B}}}^{\alpha}(\phi, \theta)=\frac{1}{2} \sum_{j, k=0}^{1} \exp [i(j \phi+k \theta)] \underline{\underline{\mathbf{B}}}_{j, k}^{\alpha} \underline{\underline{\mathbf{H}}}^{-j,-k}
$$

and

$$
\underline{\underline{\mathbf{B}}}^{\beta}(\phi, \theta)=\frac{1}{2} \sum_{j, k=0}^{1} \exp [i(j \phi+k \theta)] \underline{\underline{\mathbf{B}}}_{j, k}^{\beta} \underline{\underline{\mathbf{H}}}^{-j,-k}
$$

Substitution of the expression for $\underline{\underline{B}}_{i}^{\alpha}(2 \phi, 2 \theta) \underline{\underline{\mathbf{U}}}(\phi, \theta)$ into eq (27) yields

where

$$
\underline{\underline{\mathbf{B}}}_{i}^{\prime}(\phi, \theta)=\left[\begin{array}{llll}
\underline{\underline{\mathbf{B}}}^{+}(\phi, \theta) & \underline{\underline{\mathbf{B}}}^{+}(\phi, \bar{\theta}) & \underline{\underline{\mathbf{B}}}^{+}(\bar{\phi}, \theta) & \underline{\underline{\mathbf{B}}}^{+}(\bar{\phi}, \bar{\theta}) \\
\underline{\underline{\mathbf{B}}}^{-}(\phi, \theta) & \underline{\underline{\mathbf{B}}}^{-}(\phi, \bar{\theta}) & \underline{\underline{\mathbf{B}}}^{-}(\bar{\phi}, \theta) & \underline{\underline{\mathbf{B}}}^{-}(\bar{\phi}, \bar{\theta})
\end{array}\right]
$$

and

$$
\underline{\underline{\mathbf{B}}}^{ \pm}(\phi, \theta)=1 / \sqrt{2}\left[\underline{\underline{\mathbf{B}}}^{\alpha}(\phi, \theta) \pm \exp (-i \phi) \underline{\underline{\mathbf{B}}}^{\beta}(\phi, \theta)\right]
$$

$$
\bar{\theta}=\theta+\pi, \bar{\phi}=\phi+\pi
$$

If a diagonal valence force field is assumed for the interchain interactions

$$
\underline{\underline{\mathbf{F}}}^{\prime}(\phi, \theta)=\left(\begin{array}{llllll}
\underline{\underline{\mathbf{F}}}(\theta) & 0 & 0 & 0 & 0 & 0 \\
0 & \underline{\underline{\mathbf{F}}(\bar{\theta})} & 0 & 0 & 0 & 0 \\
0 & 0 & \underline{\underline{\mathbf{F}}}^{\circ} & 0 & 0 & 0 \\
0 & 0 & 0 & \underline{\underline{\mathbf{F}}}(\theta) & 0 & 0 \\
0 & 0 & 0 & 0 & \underline{\underline{\mathbf{F}}}(\bar{\theta}) & 0 \\
0 & 0 & 0 & 0 & 0 & \underline{\underline{\mathbf{F}}}
\end{array}\right)
$$

where $\underline{\underline{\mathbf{F}}}(\theta)$ is the isolated chain force matrix. eq (13), and $\underline{\underline{\mathbf{F}}}$ is the valence field force matrix for interchain interactions. From eqs (30-32) we have

$$
\underline{\underline{\mathbf{f}}}^{\prime}(\phi, \theta)=\left[\begin{array}{cccc}
f_{r}(\theta)+f_{i}(\phi+\theta) & f_{i}(\phi, \theta ; \phi, \bar{\theta}) & f_{i}(\phi, \theta ; \bar{\phi}, \theta) & f_{i}(\phi, \theta ; \bar{\phi}, \bar{\theta}) \\
& f_{r}(\bar{\theta})+f_{i}(\phi, \bar{\theta}) & f_{i}(\phi, \bar{\theta} ; \bar{\phi}, \theta) & f_{i}(\phi, \bar{\theta} ; \bar{\phi}, \bar{\theta}) \\
\text { c.c. } & & f_{r}(\theta)+f_{i}(\bar{\phi}, \theta) & f_{i}(\bar{\phi}, \theta ; \bar{\phi}, \bar{\theta}) \\
& & f_{r}(\bar{\theta})+f_{i}(\bar{\phi}, \bar{\theta})
\end{array}\right]
$$

where $f_{r}(\theta)$ is the symmetrized force matrix for the isolated chain [from eq $\left.(16), f_{r}(\theta)=\underline{\underline{\mathbf{B}}}(\theta) \underline{\underline{\mathbf{F}}}(\theta) \underline{\underline{\mathbf{B}}}(\theta)\right]$,

$$
f_{i}(\phi, \theta ; \phi, \bar{\theta})=\underline{\underline{\mathbf{B}}}^{+}(\phi, \theta) \underline{\underline{\mathbf{F}}}^{0} \underline{\underline{\mathbf{B}}}^{+}(\phi, \bar{\theta})+\underline{\underline{\mathbf{B}}}^{-}(\phi, \theta) \underline{\underline{\mathbf{F}}}^{0} \underline{\underline{\mathbf{B}}}^{-}(\phi, \bar{\theta})
$$


and

$$
f_{i}(\phi, \theta) \equiv f_{i}(\phi, \theta ; \phi, \theta)
$$

The vibrational secular equation for the two-dimensional lattice is

$$
\left|\underline{\underline{\mathbf{M}}}^{-1 / 2}{\underline{\underline{\mathbf{f}^{\prime}}}}^{\prime}(\phi, \theta) \underline{\underline{\mathbf{M}}}^{-1 / 2}-\omega^{2} \mathbf{I}\right|=0
$$

A relationship exists between $\underline{\underline{\mathbf{B}}}^{\alpha}(\phi, \theta)$ and $\underline{\underline{\mathbf{B}}}^{\beta}(\phi, \theta)$ which is helpful in the further simplification of $f^{\prime}(\phi, \theta)$. The quantities $\underline{\underline{\mathbf{B}}}_{j, k}^{\alpha}(\phi, \theta), B_{j, k}^{\beta}(\phi, \theta)$ which appear in eqs (29 a. b) are sums of $B$ matrices multiplied by phase factors.

and

$$
\underline{\underline{\mathbf{B}}}_{j, k}^{\alpha}(2 \phi, 2 \theta)=\sum_{l, n} \exp \left[2 i(l \phi+n \theta) \underline{\underline{\mathbf{B}}}_{j, k}^{\alpha, l, n}\right.
$$

$$
\underline{\underline{\mathbf{B}}}_{j, k}^{\beta}(2 \phi, 2 \theta)=\sum_{l, n} \exp [2 i(l \phi+n \theta)] \underline{\underline{\mathbf{B}}}_{j, k}^{\beta, l, n}
$$

where the superscripts $l, n$ label the unit cell. As the internal coordinates given by $\underline{\underline{\mathbf{B}}}_{j, k}^{\beta}(\phi, \theta)$ and $\underline{\underline{\mathbf{B}}}_{j, k}^{\alpha}(\phi, \theta)$ are equivalent it follows that

$$
\underline{\underline{\mathbf{B}}}_{j, k}^{\beta, l, n}=\underline{\underline{\mathbf{B}}}^{\alpha, l+j-1,-n}{ }_{1-1, k} \underline{\underline{\mathbf{H}}}^{-1,0}
$$

and from eq $(35 b)$

$$
\underline{\underline{\mathbf{B}}}_{j, k}^{\beta}(2 \phi, 2 \theta)=\exp [-2 i(j-1) \phi] \underline{\underline{\mathbf{B}}}_{1-j, 1-k}^{\alpha}(2 \phi,-2 \theta) \underline{\underline{\mathbf{H}}}^{-1,0} .
$$

Substituting the above expression into eq (29b) yields

and from eqs (31) and (33b)

$$
\underline{\underline{\mathrm{B}}}^{\beta}(\phi, \theta)=\exp [i(\phi+\theta)] \underline{\underline{\mathbf{B}}}^{\alpha}(\phi,-\theta) \underline{\underline{\mathbf{H}}}^{0,1}
$$

$f\left(\phi, \theta ; \phi^{\prime}, \theta^{\prime}\right)=\underline{\underline{\mathbf{B}}}^{\alpha}(\phi, \theta) \underline{\underline{\mathbf{F}}}^{0} \underline{\underline{\mathbf{B}}}^{\alpha}\left(\phi^{\prime}, \theta^{\prime}\right)+\exp \left[-i\left(\phi-\phi^{\prime}+\theta-\theta^{\prime}\right)\right] \underline{\underline{\mathbf{H}}}^{0,-1}$

$$
\underline{\underline{\mathbf{B}}}^{\alpha}(\phi,-\theta) \underline{\underline{\mathbf{F}}}^{0} \underline{\underline{B}}^{\alpha}\left(\phi^{\prime},-\theta^{\prime}\right) \underline{\underline{\mathbf{H}}}^{0,1} \text {. }
$$

The secular equation may be constructed from the $B$ and $F$ matrices for the isolated chain and the interactions between the two chains in the unit cell. Under certain conditions as shall now be shown $\underline{\underline{f}}^{\prime}(\phi, \theta)$ reduces to block form.

We assume the polymer possesses a plane of symmetry and that the two chains in the unit cell are coplanar. If the internal coordinate connecting the two chains is a bond stretch directed along the $x$ axis as in figure 1 $\underline{\underline{\mathbf{B}}}^{\alpha}(\phi,-\theta)$ is independent of $\theta$ and

$$
\underline{\underline{\mathbf{f}_{i}}}\left(\phi, \theta ; \phi^{\prime}, \theta^{\prime}\right)=\left[1+\exp \left[-i\left(\phi-\phi^{\prime}+\theta-\theta^{\prime}\right)\right]\right] \underline{\underline{\mathbf{B}}}^{\alpha}(\phi, \theta) \underline{\underline{\mathbf{F}}}^{0} \underline{\underline{B}}^{\alpha}\left(\phi^{\prime}, \theta^{\prime}\right)
$$

The quantity $\underline{\underline{\mathbf{f}}}_{i}\left(\phi, \theta ; \phi^{\prime}, \theta^{\prime}\right)=0$ if $\left(\phi^{\prime}, \theta^{\prime}\right)=(\phi+\pi, \theta)$ or $\left(\phi^{\prime}, \theta^{\prime}\right)=(\phi, \theta+\pi)$ and $\underline{\underline{\mathbf{f}}}^{\prime}(\phi, \theta)$, eq (33a) becomes upon rearrangement

$$
\stackrel{\mathbf{f}^{\prime}}{=}(\phi, \theta)=\left[\begin{array}{cccc}
f_{r}(\theta)+f_{i}(\phi, \theta) & f_{i}(\phi, \theta ; \bar{\phi}, \bar{\theta}) & 0 & 0 \\
& f_{r}(\bar{\theta})+f_{i}(\bar{\phi}, \bar{\theta}) & 0 & 0 \\
c . c . & f_{r}(\bar{\theta})+f_{i}(\phi, \bar{\theta}) & f_{i}(\phi, \bar{\theta} ; \bar{\phi}, \theta) \\
& & f_{r}(\theta)+f_{i}(\bar{\phi}, \theta)
\end{array}\right]
$$


Hence, for interchain interactions directed along the $x$ axis (perpendicular to the chain axis) the chain modes given by $\phi, \theta$ interact with those of the other chain in the unit cell given by $\phi+\pi, \theta+\pi$ with the interaction given by $2 \underline{\underline{\mathbf{B}}}_{1}^{\alpha}(\phi, \theta) \underline{\underline{\mathbf{F}}}^{0} \underline{\underline{\mathbf{B}}}_{1}^{\alpha}(\phi+\pi, \theta+\pi)$.

A dynamical matrix of the form of eq (38) is retained for interchain interactions which couple $x$ and $z$ motions if the chain possesses local $C_{2}$ symmetry about the interchain bond as in the case of the honeycomb lattice, figure 1. Consider the internal coordinates which are deformations of the angles formed by the interchain bond in figure 1. The two angles with common apex atom are equivalent because of the local $C_{2}$ symmetry. Under the two-fold screw rotation about $x$ these internal coordinates are carried into an equivalent pair in the second chain. These four internal coordinates are therefore equivalent and if the equivalent ones in $\underline{\underline{\mathbf{B}}}_{1}^{\beta}$ are renumbered it follows that

from which it can be shown that

$$
\underline{\underline{\mathbf{B}}}_{j, k}^{\beta, l, n}=\underline{\underline{\mathbf{B}}}_{1-j, 1-k}^{\alpha, j+l-1, n+k}
$$

$$
\underline{\underline{\mathbf{B}}}^{\beta}(\phi, \theta)=\exp [i(\phi-\theta)] \underline{\underline{\mathbf{B}}}^{\alpha}(\phi, \theta) \underline{\underline{\mathbf{H}}}^{-1,-1} .
$$

For in-plane internal coordinates

$$
\underline{\underline{\mathbf{B}}}^{\beta}(\phi, \theta)=-\exp [i(\phi-\theta)] \underline{\underline{\mathbf{B}}}^{\alpha}(\phi, \theta)
$$

and eq (38) follows.

\section{Example: Honeycomb Lattice}

The preceding method will now be applied to the honeycomb lattice shown in figure 1 . The in-plane intrachain internal coordinates of the honeycomb lattice are $R_{1}^{0}$. bond stretch and $R_{2}^{0}$. angle bend; one of the set of two equivalent interchain coordinates consists of $r_{\alpha}^{0}$. bond stretch and $r_{\alpha}^{2-5}$. angle deformations. Let $2 \epsilon$ be the intrachain angle. $s=\sin \epsilon, c=\cos \epsilon$ and $r, R$ the intrachain and interchain bond distances respectively. The intrachain coordinates are

$$
\left(\begin{array}{l}
R_{1}^{0} \\
R_{2}^{0}
\end{array}\right)=\left(\begin{array}{cc}
0 & 0 \\
s / r & -c / r
\end{array}\right) \underline{\mathbf{X}}_{1}^{-1}\left(\begin{array}{cc}
c & -s \\
-2 s / r & 0
\end{array}\right) \underline{\mathbf{X}}_{1}^{0}+\left(\begin{array}{cc}
-c & s \\
s / r & c / r
\end{array}\right) \underline{\mathbf{X}}_{1}^{1}
$$

and the interchain coordinates

$$
\begin{aligned}
& \left(\begin{array}{c}
r_{\alpha}^{0} \\
r_{\alpha}^{1} \\
r_{\alpha}^{2} \\
r_{\alpha}^{3} \\
r_{\alpha}^{4}
\end{array}\right)=\left(\begin{array}{cc}
0 & 0 \\
s / r & c / r \\
0 & 0 \\
0 & 0 \\
0 & 0
\end{array}\right) \underline{\mathbf{X}}_{1,1}^{0,-1}+\left(\begin{array}{cc}
0 & 0 \\
0 & 0 \\
0 & 0 \\
s / r & c / r \\
0 & 0
\end{array}\right) \underline{\mathbf{X}}_{1 ; 1}^{0,-1}+\left(\begin{array}{cc}
-1 & 0 \\
s / r & -(c / r+1 / R) \\
s / r & c / r+1 / R \\
0 & 1 / R \\
0 & -1 / R
\end{array}\right) \underline{\mathbf{X}}_{0,0}^{0,0} \\
& +\left(\begin{array}{cc}
0 & 0 \\
0 & 0 \\
-s / r & -c / r \\
0 & 0 \\
0 & 0
\end{array}\right) \underline{\mathbf{X}}_{0,1}^{0,0}+\left(\begin{array}{cc}
1 & 0 \\
0 & 1 / R \\
0 & -1 / R \\
-s / r & -(c / r+1 / R) \\
-s / r & (c / r+1 / R)
\end{array}\right) \underline{\mathbf{X}}_{1,0}^{0,0}+\left(\begin{array}{cc}
0 & 0 \\
0 & 0 \\
0 & 0 \\
0 & 0 \\
s / r & -c / r
\end{array}\right) \underline{\mathbf{X}}_{1,1}^{0,0}
\end{aligned}
$$

From eqs (12 and 13) the dynamical matrix for the isolated chain is, assuming a valence force field.

$$
\underline{\underline{\mathbf{F}}}(\theta)=\left[\begin{array}{ll}
4 \cos ^{2} \theta / 2\left(f_{r} c^{2}+4 s^{2} f_{\epsilon} / r^{2} \cos ^{2} \theta / 2\right) & 2 i s c \sin \theta\left(f_{r}-4 f_{\epsilon} / r^{2} \cos ^{2} \theta / 2\right) \\
-2 i s c \sin \theta\left(f_{r}-4 f_{\epsilon} / r^{2} \cos ^{2} \theta / 2\right) & 4 f_{r} s^{2} \sin ^{2} \theta / 2+4 f_{\epsilon} / r^{2} c^{2} \sin ^{2} \theta
\end{array}\right]
$$


where $f_{r}, f_{\epsilon}$ are the bond stretch and angle deformation force constants respectively. The interaction matrices are found through eqs (28), (29a), and (37) to be

$$
\begin{aligned}
& f(\phi, \theta ; \phi, \theta)=\left[\begin{array}{lr}
2 f_{R} \sin ^{2}(\phi / 2)+8 f_{\delta} s^{2} / r^{2} \cos ^{2} \theta / 2 & -4 i f_{\delta} s / r \sin (\theta)\left[c / r+\cos ^{2}(\phi / 2) / R\right] \\
4 i f_{\delta} s / r \sin (\theta)\left[c / r+\cos ^{2}(\phi / 2) / R\right] & 8 f_{\delta}\left(c^{2} / r^{2} \sin ^{2}(\theta / 2)+1 / R_{1}^{2} \cos ^{2}(\phi / 2)+2 c / r R\right. \\
\left.\cos ^{2}(\phi / 2) \sin ^{2}(\theta / 2)\right)
\end{array}\right] \\
& f(\phi, \theta ; \bar{\phi}, \bar{\theta})=\left[\begin{array}{lr}
i f_{R} \sin \phi & -2 f_{\delta} s / r R \sin \phi \sin \theta \\
-2 \mathrm{f}_{\delta} s / r R \sin \phi \sin \theta & -4 i f_{\delta} / R \sin (\phi)(1 / R+c / r)
\end{array}\right]
\end{aligned}
$$

where $f_{R}, f_{\delta}$ are the interchain force constants for bond stretch and angle bend respectively. From $f(\phi, \theta ; \phi, \theta)$ and $f(\phi, \theta ; \bar{\phi}, \bar{\theta})$ the first block of $\underline{\underline{\mathbf{f}}}^{\prime}(\phi, \theta)$, eq (38), may be constructed. The resultant secular equation may be solved for values of $0 \leqslant \phi \leqslant \pi / 2$ and $0 \leqslant \theta \leqslant \pi$ to obtain the phonon dispersion curves over the $z$-extended zone. The variation of phonon energies with $\underline{\mathbf{k}}_{z}$ for a particular value of $\underline{\mathbf{k}}_{x}$ are found by folding the phonon dispersion curves about $\theta=90^{\circ}$.

\section{Summary}

Utilizing the unit cell symmetry, the dynamical matrix (eq (33a)) of the antiparallel chain lattice has been derived. The elements of this matrix are expressed in terms of the dynamical matrix for the isolated chain and the $B$ and $F$ matrices for the interchain coordinates. The dynamical matrix of the isolated chain and hence that of the lattice may be determined by specifying the internal coordinates of one chemical repeat unit. It was found that with certain types of interchain forces the dynamical matrix of the antiparallel chain lattice may be reduced to block form for arbitrary values of the intrachain and interchain phases. All normal modes may be determined by solving one of the two blocks of resulting secular equation for $0 \leqslant \phi \leqslant \pi / 2$ and $0 \leqslant \theta \leqslant \pi$. The reduction in the dimensionality of the secular equation with certain types of interchain forces can lead to a significant reduction in computation time especially for more complicated homopolymers such as polyglycine.

The methods used to derive the dynamical matrix of the antiparallel chain lattice may also be applied to other polymeric crystal structures.

\section{References}

[1] Peticolas, W. L., Hibler, G. W., Lippert, J. L., Peterlin, A., and Olf, H., Appl. Phys. Let. 18, 87 (1971).

[2] Itoh, K., Nakahara, T., and Shimanouchi, T., Biopolymers 6, 1759 (1968).

[3] Fanconi, B., and Peticolas, W. L., Biopolymers (in press).

[4] Fukushima, K., Ideguchi, Y., and Miyazawa, T., Bull. Chem. Soc. Japan 36, 1301 (1963).

[5] Gupta, V. D., Trevino, S., and Boutin, H., J. Chem. Phys. 48, 3008 (1968).

[6] Small, E. W., Fanconi, B., and Peticolas, W. L., J. Chem. Phys. 52, 4369 (1970).

[7] Fanconi, B., Small, E. W., and Peticolas, W. L., Biopolymers 10 , 1277 (1971).

[8] Miyazawa, T., J. Chem. Phys. 32, 1647 (1960).

[9] Gribov, L. A., Opt. Spectrosc. 29, 465 (1970).

[10] Gupta, V. D., Gupta, A. K., and Krishnan, M. V., Chem. Phys. Letters 6, 317 (1970).

[11] Wilson, E. B., Jr., J. Chem. Phys. 7, 1047 (1939).

[12] Higgs, P. W., Proc. Roy. Soc. (London) A220, 472 (1953).

[13] Miyazawa, T., J. Chem. Phys. 35, 693 (1961); 38, 2709 (1963).

[14] Piseri, L., and Zerbi, G., J. Mol. Spectry. 26, 254 (1968).

[15] Dean, P., and Bacon, M. D., Proc. Roy. Soc. (London), Ser. A283, 64 (1964). 